\title{
Subordination and superordination of certain linear operator on meromorphic functions
}

\begin{abstract}
Using the methods of differential subordination and superordination, sufficient conditions are determined on the differential linear operator of meromorphic functions in the punctured unit disk to obtain, respectively, the best dominant and the best subordinant. New sandwich-type results are also obtained.
\end{abstract}

1. Introduction. Let $H(U)$ be the class of functions analytic in $U=\{z$ : $z \in C$ and $|z|<1\}$ and $H[a, n]$ be the subclass of $H(U)$ consisting of functions of the form $f(z)=a+a_{n} z^{n}+a_{n+1} z^{n+1}+\ldots$, with $H=H[1,1]$. Let $f$ and $F$ be members of $H(U)$. The function $f$ is said to be subordinate to $F$, or $F$ is said to be superordinate to $f$, if there exists a function $\omega$ analytic in $U$ with $\omega(0)=0$ and $|\omega(z)|<1(z \in U)$, such that $f(z)=$ $F(\omega(z))$. In such a case we write $f(z) \prec F(z)$. If $F$ is univalent, then $f(z) \prec F(z)$ if and only if $f(0)=F(0)$ and $f(U) \subset F(U)$ (see [5] and [6]).

Denote by $Q$ the set of all functions $q(z)$ that are analytic and injective on $\bar{U} \backslash E(q)$ where

$$
E(q)=\left\{\zeta \in \partial U: \lim _{z \rightarrow \zeta} q(z)=\infty\right\}
$$

2000 Mathematics Subject Classification. 30C45.

Key words and phrases. Analytic function, linear operator, Hadamard product, differential subordination, superordination. 
and are such that $q^{\prime}(\zeta) \neq 0$ for $\zeta \in \partial U \backslash E(q)$. Further let the subclass of $Q$ for which $q(0)=a$ be denoted by $Q(a)$ and $Q(1) \equiv Q_{1}$.

In order to prove our results, we shall make use of the following classes of admissible functions.

Definition 1 ([5, Definition 2.3a, p. 27]). Let $\Omega$ be a set in $\mathbb{C}, q \in Q$ and $n$ be a positive integer. The class of admissible functions $\Psi_{n}[\Omega, q]$ consists of those functions $\psi: \mathbb{C}^{3} \times U \rightarrow \mathbb{C}$ which satisfy the admissibility condition:

$$
\psi(r, s, t ; z) \notin \Omega
$$

whenever $r=q(\zeta), s=k \zeta q^{\prime}(\zeta)$,

$$
\Re\left\{\frac{t}{s}+1\right\} \geq k \Re\left\{1+\frac{\zeta q^{\prime \prime}(\zeta)}{q^{\prime}(\zeta)}\right\},
$$

where $z \in U, \zeta \in \partial U \backslash E(q)$ and $k \geq n$. We write $\Psi_{1}[\Omega, q]$ as $\Psi[\Omega, q]$.

In particular, if

$$
q(z)=M \frac{M z+a}{M+\bar{a} z} \quad(M>0,|a|<M),
$$

then $q(U)=U_{M}=\{w:|w|<M\}, q(0)=a, E(q)=\emptyset$ and $q \in Q(a)$. In this case, we set $\Psi_{n}[\Omega, M, a]=\Psi_{n}[\Omega, q]$, and in the special case when the set $\Omega=U_{M}$, the class is simply denoted by $\Psi_{n}[M, a]$.

Definition 2 ([6, Definition 3, p. 817]). Let $\Omega$ be a set in $\mathbb{C}, q(z) \in H[a, n]$ with $q^{\prime}(z) \neq 0$. The class of admissible functions $\Psi_{n}^{\prime}[\Omega, q]$ consists of those functions $\psi: \mathbb{C}^{3} \times \bar{U} \rightarrow \mathbb{C}$ which satisfy the admissibility condition:

$$
\psi(r, s, t ; \zeta) \in \Omega
$$

whenever $r=q(z), s=\frac{z q^{\prime}(z)}{m}$,

$$
\Re\left\{\frac{t}{s}+1\right\} \leq \frac{1}{m} \Re\left\{1+\frac{z q^{\prime \prime}(z)}{q^{\prime}(z)}\right\},
$$

where $z \in U, \zeta \in \partial U$ and $m \geq n \geq 1$. In particular, we write $\Psi_{1}^{\prime}[\Omega, q]$ as $\Psi^{\prime}[\Omega, q]$.

In our investigation we need the following lemmas which are proved by Miller and Mocanu ([5] and [6]).

Lemma 1 ([5, Theorem 2.3b, p. 28]). Let $\psi \in \Psi_{n}[\Omega, q]$ with $q(0)=a$. If the analytic function $g(z)=a+a_{n} z^{n}+a_{n+1} z^{n+1}+\ldots$ satisfies

$$
\psi\left(g(z), z g^{\prime}(z), z^{2} g^{\prime \prime}(z) ; z\right) \in \Omega,
$$

then $g(z) \prec q(z)$. 
Lemma 2 ([6, Theorem 1, p. 818]). Let $\psi \in \Psi_{n}^{\prime}[\Omega, q]$ with $q(0)=a$. If $g(z) \in Q(a)$ and

$$
\psi\left(g(z), z g^{\prime}(z), z^{2} g^{\prime \prime}(z) ; z\right)
$$

is univalent in $U$, then

$$
\Omega \subset\left\{\psi\left(g(z), z g^{\prime}(z), z^{2} g^{\prime \prime}(z) ; z\right): z \in U\right\},
$$

implies $q(z) \prec g(z)$.

Let $\sum_{p}$ denote the class of all $p$-valent functions of the form:

(1.1) $f(z)=\frac{1}{z^{p}}+\sum_{k=1-p}^{\infty} a_{k} z^{k} \quad\left(p \in \mathbb{N}=\{1,2,3, \ldots\} ; z \in U^{*}=U \backslash\{0\}\right)$.

For two functions $f$ given by (1.1) and $g$ given by

$$
g(z)=\frac{1}{z^{p}}+\sum_{k=1-p}^{\infty} b_{k} z^{k},
$$

the Hadamard product (or convolution) of $f$ and $g$ is defined by

$$
(f * g)(z)=\frac{1}{z^{p}}+\sum_{k=1-p}^{\infty} a_{k} b_{k} z^{k}=(g * f)(z) .
$$

For a function $f$ in the class $\sum_{p}$ given by (1.1), we define a linear operator $D_{\lambda, p}^{n}: \sum_{p} \rightarrow \sum_{p}$ as follows:

$$
\begin{gathered}
D_{\lambda, p}^{0} f(z)=f(z), \\
D_{\lambda, p}^{1} f(z)=D_{\lambda}(f(z))=(1-\lambda) f(z)+\lambda \frac{\left(z^{p+1} f(z)\right)^{\prime}}{z^{p}} \\
=\frac{1}{z^{p}}+\sum_{k=1-p}^{\infty}[1+\lambda(k+p)] a_{k} z^{k} \quad(\lambda \geq 0 ; p \in \mathbb{N}), \\
D_{\lambda, p}^{2} f(z)=D_{\lambda}\left(D_{\lambda, p}^{1} f(z)\right)=(1-\lambda) D_{\lambda, p}^{1} f(z)+\lambda \frac{\left(z^{p+1} D_{\lambda, p}^{1} f(z)\right)^{\prime}}{z^{p}} \\
=\frac{1}{z^{p}}+\sum_{k=1-p}^{\infty}[1+\lambda(k+p)]^{2} a_{k} z^{k} \quad(\lambda \geq 0 ; p \in \mathbb{N}),
\end{gathered}
$$


and (in general)

$$
\begin{aligned}
D_{\lambda, p}^{n} f(z) & =D_{\lambda}\left(D_{\lambda, p}^{n-1} f(z)\right) \\
& =(1-\lambda) D_{\lambda, p}^{n-1} f(z)+\lambda \frac{\left(z^{p+1} D_{\lambda, p}^{n-1} f(z)\right)^{\prime}}{z^{p}} \\
& =\frac{1}{z^{p}}+\sum_{k=1-p}^{\infty}[1+\lambda(k+p)]^{n} a_{k} z^{k} \\
& \left(\lambda \geq 0 ; p \in \mathbb{N} ; n \in \mathbb{N}_{0}=\mathbb{N} \cup\{0\}\right) .
\end{aligned}
$$

It is easily verified from (1.4) that

$$
\begin{aligned}
\lambda z\left(D_{\lambda, p}^{n} f(z)\right)^{\prime}= & (1-\lambda) D_{\lambda, p}^{n+1} f(z)-(1+\lambda p) D_{\lambda, p}^{n} f(z) \\
& \left(\lambda>0 ; p \in \mathbb{N} ; n \in \mathbb{N}_{0}\right) .
\end{aligned}
$$

We note that:

(i) The operator $D_{1, p}^{n}=D_{p}^{n}$ was introduced and studied by Aouf and Hossen [2], Liu and Owa [3], Liu and Srivastava [4], Srivastava and Patel [7];

(ii) The operator $D_{1,1}^{n}=D^{n}$ was considered and studied by Uralegaddi and Somanatha [8].

In the present paper, by making use of the differential subordination and superordination results of Miller and Mocanu [5, Theorem 2.3b, p. 28] and $[6$, Theorem 1, p. 818], certain classes of admissible functions are determined so that subordination as well as superordination implications of functions associated with the linear operator $D_{\lambda, p}^{n}$ hold. Ali et al. [1] have considered a similar problem for Liu-Srivastava linear operator on meromorphic functions. Additionally, several differential sandwich-type results are obtained.

2. Subordination results involving the linear operator $D_{\lambda, p}^{n}$. Unless otherwise mentioned, we assume throughout this paper that $\lambda>0, p \in \mathbb{N}$ and $n \in \mathbb{N}_{0}$. The following class of admissible functions is required in our first result.

Definition 3. Let $\Omega$ be a set in $\mathbb{C}$ and $q(z) \in Q_{1} \cap H$. The class of admissible functions $\Phi_{D}[\Omega, q]$ consists of those functions $\phi: \mathbb{C}^{3} \times U \rightarrow \mathbb{C}$ which satisfy the admissibility condition

$$
\phi(u, v, w ; z) \notin \Omega
$$

whenever $u=q(\zeta), v=k \lambda \zeta q^{\prime}(\zeta)+q(\zeta)$,

$$
\Re\left\{\frac{w-2 v+u}{\lambda(v-u)}\right\} \geq k \Re\left\{1+\frac{\zeta q^{\prime \prime}(\zeta)}{q^{\prime}(\zeta)}\right\},
$$

where $z \in U, \zeta \in \partial U \backslash E(q)$ and $k \geq 1$. 
Theorem 1. Let $\phi \in \Phi_{D}[\Omega, q]$. If $f(z) \in \sum_{p}$ satisfies

$$
\left\{\phi\left(z^{p} D_{\lambda, p}^{n} f(z), z^{p} D_{\lambda, p}^{n+1} f(z), z^{p} D_{\lambda, p}^{n+2} f(z) ; z\right): z \in U\right\} \subset \Omega,
$$

then

$$
z^{p} D_{\lambda, p}^{n} f(z) \prec q(z) .
$$

Proof. Define the analytic function $g(z)$ in $U$ by

$$
g(z)=z^{p} D_{\lambda, p}^{n} f(z) .
$$

From (1.5) and (2.2), we have

$$
z^{p} D_{\lambda, p}^{n+1} f(z)=g(z)+\lambda z g^{\prime}(z) .
$$

Further computations show that

$$
z^{p} D_{\lambda, p}^{n+2} f(z)=\lambda^{2} z^{2} g^{\prime \prime}(z)+\lambda(2+\lambda) z g^{\prime}(z)+g(z) .
$$

Define the transformations from $\mathbb{C}^{3}$ to $\mathbb{C}$ by

$$
u(r, s, t)=r, v(r, s, t)=r+\lambda s, w(r, s, t)=r+\lambda(2+\lambda) s+\lambda^{2} t .
$$

Let

$$
\psi(r, s, t ; z)=\phi(u, v, w ; z)=\phi\left(r, r+\lambda s, r+\lambda(2+\lambda) s+\lambda^{2} t ; z\right) .
$$

The proof shall make use of Lemma 1. Using equations (2.2)-(2.4), and from (2.6), we obtain

$$
\begin{aligned}
& \psi\left(g(z), z g^{\prime}(z), z^{2} g^{\prime \prime}(z) ; z\right) \\
& \quad=\phi\left(z^{p} D_{\lambda, p}^{n} f(z), z^{p} D_{\lambda, p}^{n+1} f(z), z^{p} D_{\lambda, p}^{n+2} f(z) ; z\right) .
\end{aligned}
$$

Hence (2.1) becomes

$$
\psi\left(g(z), z g^{\prime}(z), z^{2} g^{\prime \prime}(z) ; z\right) \in \Omega .
$$

The proof is completed, if it can be shown that the admissibility condition for $\phi \in \Phi_{D}[\Omega, q]$ is equivalent to the admissibility condition for $\psi$ as given in Definition 1. Note that

$$
\frac{t}{s}+1=\frac{w-2 v+u}{\lambda(v-u)},
$$

and hence $\psi \in \Psi[\Omega, q]$. By Lemma 1 ,

$$
g(z) \prec q(z) \quad \text { or } \quad z^{p} D_{\lambda, p}^{n} f(z) \prec q(z) \text {. }
$$

If $\Omega \neq \mathbb{C}$ is a simply connected domain, then $\Omega=h(U)$ for some conformal mapping $h(z)$ of $U$ onto $\Omega$. In this case the class $\Phi_{D}[h(U), q]$ is written as $\Phi_{D}[h, q]$.

The following result is an immediate consequence of Theorem 1. 
Theorem 2. Let $\phi \in \Phi_{D}[h, q]$ with $q(0)=1$. If $f(z) \in \sum_{p}$ satisfies

$$
\phi\left(z^{p} D_{\lambda, p}^{n} f(z), z^{p} D_{\lambda, p}^{n+1} f(z), z^{p} D_{\lambda, p}^{n+2} f(z) ; z\right) \prec h(z),
$$

then

$$
z^{p} D_{\lambda, p}^{n} f(z) \prec q(z) .
$$

Our next result is an extension of Theorem 1 to the case where the behavior of $q(z)$ on $\partial U$ is not known.

Corollary 1. Let $\Omega \subset \mathbb{C}$ and let $q(z)$ be univalent in $U, q(0)=1$. Let $\phi \in \Phi_{D}\left[\Omega, q_{\rho}\right]$ for some $\rho \in(0,1)$, where $q_{\rho}(z)=q(\rho z)$. If $f(z) \in \sum_{p}$ and

$$
\phi\left(z^{p} D_{\lambda, p}^{n} f(z), z^{p} D_{\lambda, p}^{n+1} f(z), z^{p} D_{\lambda, p}^{n+2} f(z) ; z\right) \in \Omega,
$$

then

$$
z^{p} D_{\lambda, p}^{n} f(z) \prec q(z) .
$$

Proof. Theorem 1 yields $z^{p} D_{\lambda, p}^{n} f(z) \prec q_{\rho}(z)$. The result is now deduced from $q_{\rho}(z) \prec q(z)$.

Theorem 3. Let $h(z)$ and $q(z)$ be univalent in $U$, with $q(0)=1$ and set $q_{\rho}(z)=q(\rho z)$ and $h_{\rho}(z)=h(\rho z)$. Let $\phi: \mathbb{C}^{3} \times U \rightarrow \mathbb{C}$ satisfy one of the following conditions:

(1) $\phi \in \Phi_{D}\left[h, q_{\rho}\right]$, for some $\rho \in(0,1)$, or

(2) there exists $\rho_{0} \in(0,1)$ such that $\phi \in \Phi_{D}\left[h_{\rho}, q_{\rho}\right]$, for all $\rho \in\left(\rho_{0}, 1\right)$.

If $f(z) \in \sum_{p}$ satisfies (2.8), then

$$
z^{p} D_{\lambda, p}^{n} f(z) \prec q(z) .
$$

Proof. The proof is similar to the proof of [4, Theorem 2.3d, p. 30] and is therefore omitted.

The next theorem yields the best dominant of the differential subordination (2.8).

Theorem 4. Let $h(z)$ be univalent in $U$ and $\phi: \mathbb{C}^{3} \times U \rightarrow \mathbb{C}$. Suppose that the differential equation

(2.9) $\phi\left(g(z), g(z)+\lambda z g^{\prime}(z), \lambda^{2} z^{2} g^{\prime \prime}(z)+\lambda(2+\lambda) z g^{\prime}(z)+g(z) ; z\right)=h(z)$

has a solution $q(z)$ with $q(0)=1$ and satisfies one of the following conditions:

(1) $q(z) \in Q_{1}$ and $\phi \in \Phi_{D}[h, q]$,

(2) $q(z)$ is univalent in $U$ and $\phi \in \Phi_{D}\left[h, q_{\rho}\right]$, for some $\rho \in(0,1)$, or

(3) $q(z)$ is univalent in $U$ and there exists $\rho_{0} \in(0,1)$ such that $\phi \in$ $\Phi_{D}\left[h_{\rho}, q_{\rho}\right]$, for all $\rho \in\left(\rho_{0}, 1\right)$. 
If $f(z) \in \sum_{p}$ satisfies (2.8), then

$$
z^{p} D_{\lambda, p}^{n} f(z) \prec q(z)
$$

and $q(z)$ is the best dominant.

Proof. Following the same arguments in [4, Theorem 2.3e, p. 31], we deduce that $q(z)$ is a dominant from Theorems 2 and 3. Since $q(z)$ satisfies (2.9), it is also a solution of (2.8) and therefore $q(z)$ will be dominated by all dominants. Hence $q(z)$ is the best dominant.

In the particular case $q(z)=1+M z, M>0$, and in view of Definition 3, the class of admissible functions $\Phi_{D}[\Omega, q]$, denoted by $\Phi_{D}[\Omega, M]$, is described below.

Definition 4. Let $\Omega$ be a set in $\mathbb{C}$ and $M>0$. The class of admissible functions $\Phi_{D}[\Omega, M]$ consists of those functions $\phi: \mathbb{C}^{3} \times U \rightarrow \mathbb{C}$ such that

$$
\phi\left(1+M e^{i \theta}, 1+(1+\lambda k) M e^{i \theta}, 1+\lambda^{2} L+[1+\lambda(2+\lambda) k] M e^{i \theta} ; z\right) \notin \Omega
$$

whenever $z \in U, \theta \in \mathbb{R}, \Re\left(L e^{-i \theta}\right) \geq(k-1) k M$ for all real $\theta, \lambda>0, p \in \mathbb{N}$ and $k \geq 1$.

Corollary 2. Let $\phi \in \Phi_{D}[\Omega, M]$. If $f(z) \in \sum_{p}$ satisfies

$$
\phi\left(z^{p} D_{\lambda, p}^{n} f(z), z^{p} D_{\lambda, p}^{n+1} f(z), z^{p} D_{\lambda, p}^{n+2} f(z) ; z\right) \in \Omega,
$$

then

$$
\left|z^{p} D_{\lambda, p}^{n} f(z)-1\right|<M
$$

In the special case $\Omega=q(U)=\{\omega:|\omega-1|<M\}$, the class $\Phi_{D}[\Omega, M]$ is simply denoted by $\Phi_{D}[M]$. Corollary 2 can now be written the following form:

Corollary 3. Let $\phi \in \Phi_{D}[M]$. If $f(z) \in \sum_{p}$ satisfies

$$
\left|\phi\left(z^{p} D_{\lambda, p}^{n} f(z), z^{p} D_{\lambda, p}^{n+1} f(z), z^{p} D_{\lambda, p}^{n+2} f(z) ; z\right)-1\right|<M,
$$

then

$$
\left|z^{p} D_{\lambda, p}^{n} f(z)-1\right|<M
$$

Corollary 4. If $M>0$ and $f(z) \in \sum_{p}$ satisfies

$$
\left|z^{p} D_{\lambda, p}^{n+1} f(z)-1\right|<M,
$$

then

$$
\left|z^{p} D_{\lambda, p}^{n} f(z)-1\right|<M
$$

Proof. This follows from Corollary 3 by taking $\phi(u, v, w ; z)=v=1+$ $(1+\lambda k) M e^{i \theta}$. 
Corollary 5. If $M>0$ and $f(z) \in \sum_{p}$ satisfies

$$
\left|z^{p} D_{\lambda, p}^{n+1} f(z)-z^{p} D_{\lambda, p}^{n} f(z)\right|<M
$$

then

$$
\left|z^{p} D_{\lambda, p}^{n} f(z)-1\right|<M .
$$

Proof. Let $\phi(u, v, w ; z)=v-u$ and $\Omega=h(U)$ where $h(z)=M z, M>$ 0 . To use Corollary 2 , we need to show that $\phi \in \Phi_{D}[\Omega, M]$, that is, the admissibility condition (2.10) is satisfied. This follows from

$$
\begin{aligned}
& \left|\phi\left(1+M e^{i \theta}, 1+(1+\lambda k) M e^{i \theta}, 1+\lambda^{2} L+[1+\lambda(2+\lambda) k] M e^{i \theta} ; z\right)\right| \\
& \quad=M \lambda k \geq M \lambda
\end{aligned}
$$

whenever $z \in U, \theta \in \mathbb{R}, \lambda>0, p \in \mathbb{N}$ and $k \geq 1$. The required result now follows from Corollary 2.

Theorem 4 shows that the result is sharp. The differential equation

$$
\lambda z q^{\prime}(z)=\lambda M z
$$

has a univalent solution $q(z)=1+M z$. It follows from Theorem 4 that $q(z)=1+M z$ is the best dominant.

Next, let us note that

$$
D_{1, p}^{0} f(z)=f(z)
$$

and

$$
D_{1, p}^{1} f(z)=\frac{\left(z^{p+1} f(z)\right)^{\prime}}{z^{p}}=z f^{\prime}(z)+(1+p) f(z) .
$$

By taking $n=0$ and $\lambda=1$, Corollary 5 shows that for $f \in \sum_{p}$, if $z^{p}\left[z f^{\prime}(z)+p f(z)\right] \prec M z$, then $z^{p} f(z) \prec 1+M z$.

Definition 5. Let $\Omega$ be a set in $\mathbb{C}$ and $q(z) \in Q_{1} \cap H$. The class of admissible functions $\Phi_{D, 1}[\Omega, q]$ consists of those functions $\phi: \mathbb{C}^{3} \times U \rightarrow \mathbb{C}$ which satisfy the admissibility condition:

$$
\phi(u, v, w ; z) \notin \Omega
$$

whenever $u=q(\zeta), v=q(\zeta)+\lambda \frac{k \zeta q^{\prime}(\zeta)}{q(\zeta)}(q(\zeta) \neq 0)$,

$$
\Re\left\{\frac{v w-3 u v+2 u^{2}}{\lambda(v-u)}\right\} \geq k \Re\left\{1+\frac{\zeta q^{\prime \prime}(\zeta)}{q^{\prime}(\zeta)}\right\},
$$

where $z \in U, \zeta \in \partial U \backslash E(q)$ and $k \geq 1$.

Theorem 5. Let $\phi \in \Phi_{D, 1}[\Omega, q]$. If $f(z) \in \sum_{p}$ satisfies

$$
\left\{\phi\left(\frac{D_{\lambda, p}^{n+1} f(z)}{D_{\lambda, p}^{n} f(z)}, \frac{D_{\lambda, p}^{n+2} f(z)}{D_{\lambda, p}^{n+1} f(z)}, \frac{D_{\lambda, p}^{n+3} f(z)}{D_{\lambda, p}^{n+2} f(z)} ; z\right): z \in U\right\} \subset \Omega,
$$


then

$$
\frac{D_{\lambda, p}^{n+1} f(z)}{D_{\lambda, p}^{n} f(z)} \prec q(z) .
$$

Proof. Define an analytic function $g(z)$ in $U$ by

$$
g(z)=\frac{D_{\lambda, p}^{n+1} f(z)}{D_{\lambda, p}^{n} f(z)} .
$$

Defferentiating (2.12) logarithmically with respect to $z$, we obtain

$$
\frac{z g^{\prime}(z)}{g(z)}=\frac{z\left(D_{\lambda, p}^{n+1} f(z)\right)^{\prime}}{D_{\lambda, p}^{n+1} f(z)}-\frac{z\left(D_{\lambda, p}^{n} f(z)\right)^{\prime}}{D_{\lambda, p}^{n} f(z)} .
$$

By making use of (1.5) in (2.13), we get

$$
\frac{D_{\lambda, p}^{n+2} f(z)}{D_{\lambda, p}^{n+1} f(z)}=g(z)+\lambda \frac{z g^{\prime}(z)}{g(z)} .
$$

Differentiating (2.14) logarithmically with respect to $z$, further computations show that

$$
\begin{aligned}
\frac{D_{\lambda, p}^{n+3} f(z)}{D_{\lambda, p}^{n+2} f(z)}=g(z)+\lambda \frac{z g^{\prime}(z)}{g(z)} & \\
& +\lambda \frac{z g^{\prime}(z)+\lambda\left[\frac{z g^{\prime}(z)}{g(z)}-\left(\frac{z g^{\prime}(z)}{g(z)}\right)^{2}+\frac{z^{2} g^{\prime \prime}(z)}{g(z)}\right]}{g(z)+\lambda \frac{z g^{\prime}(z)}{g(z)}}
\end{aligned}
$$

Define the transformations from $\mathbb{C}^{3}$ to $\mathbb{C}$ by

$$
\begin{aligned}
& u(r, s, t)=r, v(r, s, t)=r+\lambda \frac{s}{r}, \\
& w(r, s, t)=r+\lambda \frac{s}{r}+\lambda \frac{s+\lambda\left[\frac{s}{r}-\left(\frac{s}{r}\right)^{2}+\frac{t}{r}\right]}{r+\lambda \frac{s}{r}} .
\end{aligned}
$$

Let

$$
\begin{aligned}
\psi(r, s, t ; z) & =\phi(u, v, w ; z) \\
& =\phi\left(r, r+\lambda \frac{s}{r}, r+\lambda \frac{s}{r}+\lambda \frac{s+\lambda\left[\frac{s}{r}-\left(\frac{s}{r}\right)^{2}+\frac{t}{r}\right]}{r+\lambda \frac{s}{r}} ; z\right) .
\end{aligned}
$$


Using equations (2.12), (2.14), (2.15), and from (2.17), it follows that

$$
\begin{aligned}
\psi\left(g(z), z g^{\prime}(z), z^{2} g^{\prime \prime}(z) ; z\right) & \\
= & \phi\left(\frac{D_{\lambda, p}^{n+1} f(z)}{D_{\lambda, p}^{n} f(z)}, \frac{D_{\lambda, p}^{n+2} f(z)}{D_{\lambda, p}^{n+1} f(z)}, \frac{D_{\lambda, p}^{n+3} f(z)}{D_{\lambda, p}^{n+2} f(z)} ; z\right) .
\end{aligned}
$$

Hence (2.11) implies

$$
\psi\left(g(z), z g^{\prime}(z), z^{2} g^{\prime \prime}(z) ; z\right) \in \Omega .
$$

The proof is completed if it can be shown that the admissibility condition for $\phi \in \Phi_{D, 1}[\Omega, q]$ is equivalent to the admissibility condition for $\psi$ as given in Definition 1. Note that

$$
\frac{t}{s}+1=\frac{v w-3 u v+2 u^{2}}{\lambda(v-u)} .
$$

Hence $\psi \in \Psi[\Omega, q]$ and by Lemma 1 ,

$$
g(z) \prec q(z) \quad \text { or } \quad \frac{D_{\lambda, p}^{n+1} f(z)}{D_{\lambda, p}^{n} f(z)} \prec q(z) .
$$

In the case $\Omega \neq \mathbb{C}$ is a simply connected domain with $\Omega=h(U)$ for some conformal mapping $h(z)$ of $U$ onto $\Omega$, the class $\Phi_{D, 1}[h(U), q]$ is written as $\Phi_{D, 1}[h, q]$.

The following result is an immediate consequence of Theorem 5 .

Theorem 6. Let $\phi \in \Phi_{D, 1}[h, q]$ with $q(0)=1$. If $f(z) \in \sum_{p}$ satisfies

$$
\phi\left(\frac{D_{\lambda, p}^{n+1} f(z)}{D_{\lambda, p}^{n} f(z)}, \frac{D_{\lambda, p}^{n+2} f(z)}{D_{\lambda, p}^{n+1} f(z)}, \frac{D_{\lambda, p}^{n+3} f(z)}{D_{\lambda, p}^{n+2} f(z)} ; z\right) \prec h(z),
$$

then

$$
\frac{D_{\lambda, p}^{n+1} f(z)}{D_{\lambda, p}^{n} f(z)} \prec q(z) .
$$

In the particular case $q(z)=1+M z, M>0$, the class of admissible functions $\Phi_{D, 1}[\Omega, q]$, is simply denoted by $\Phi_{D, 1}[\Omega, M]$.

Definition 6. Let $\Omega$ be a set in $\mathbb{C}$ and $M>0$. The class of admissible functions $\Phi_{D, 1}[\Omega, M]$ consists of those functions $\phi: \mathbb{C}^{3} \times U \rightarrow \mathbb{C}$ such that

$$
\begin{aligned}
& \phi\left(1+M e^{i \theta}, 1+\frac{\lambda k+1+M e^{i \theta}}{1+M e^{i \theta}} M e^{i \theta}, 1+\frac{\lambda k+1+M e^{i \theta}}{1+M e^{i \theta}} M e^{i \theta}\right. \\
& \left.+\frac{\lambda\left(M+e^{-i \theta}\right)\left[\lambda L e^{-i \theta}+k M\left(1+\lambda+M e^{i \theta}\right)\right]-\lambda^{2} M^{2} k^{2}}{\left(M+e^{-i \theta}\right)\left(2 M+\lambda k M+e^{-i \theta}+M^{2} e^{i \theta}\right)} ; z\right) \notin \Omega
\end{aligned}
$$

whenever $z \in U, \theta \in \mathbb{R}, \Re\left(L e^{-i \theta}\right) \geq(k-1) k M$ for all real $\theta, \lambda>0$ and $k \geq 1$. 
Corollary 6. Let $\phi \in \Phi_{D, 1}[\Omega, M]$. If $f(z) \in \sum_{p}$ satisfies

$$
\phi\left(\frac{D_{\lambda, p}^{n+1} f(z)}{D_{\lambda, p}^{n} f(z)}, \frac{D_{\lambda, p}^{n+2} f(z)}{D_{\lambda, p}^{n+1} f(z)}, \frac{D_{\lambda, p}^{n+3} f(z)}{D_{\lambda, p}^{n+2} f(z)} ; z\right) \in \Omega,
$$

then

$$
\left|\frac{D_{\lambda, p}^{n+1} f(z)}{D_{\lambda, p}^{n} f(z)}-1\right|<M \quad(z \in U) .
$$

In the special case $\Omega=q(U)=\{\omega:|\omega-1|<M\}$, the class $\Phi_{D, 1}[\Omega, M]$ is denoted by $\Phi_{D, 1}[M]$, and Corollary 6 takes the following form:

Corollary 7. Let $\phi \in \Phi_{D, 1}[M]$. If $f(z) \in \sum_{p}$ satisfies

$$
\left|\phi\left(\frac{D_{\lambda, p}^{n+1} f(z)}{D_{\lambda, p}^{n} f(z)}, \frac{D_{\lambda, p}^{n+2} f(z)}{D_{\lambda, p}^{n+1} f(z)}, \frac{D_{\lambda, p}^{n+3} f(z)}{D_{\lambda, p}^{n+2} f(z)} ; z\right)-1\right|<M,
$$

then

$$
\left|\frac{D_{\lambda, p}^{n+1} f(z)}{D_{\lambda, p}^{n} f(z)}-1\right|<M .
$$

Corollary 8. Let $M>0$. If $f(z) \in \sum_{p}$ satisfies

$$
\left|\frac{D_{\lambda, p}^{n+2} f(z)}{D_{\lambda, p}^{n+1} f(z)}-\frac{D_{\lambda, p}^{n+1} f(z)}{D_{\lambda, p}^{n} f(z)}\right|<\frac{\lambda M}{1+M}
$$

then

$$
\left|\frac{D_{\lambda, p}^{n+1} f(z)}{D_{\lambda, p}^{n} f(z)}-1\right|<M
$$

Proof. This follows from Corollary 6 by taking $\phi(u, v, w ; z)=v-u$ and $\Omega=h(U)$, where $h(z)=\frac{\lambda M}{1+M} z, M>0$. To use Corollary 6 we need to show that $\phi \in \Phi_{D, 1}[M]$, i.e., the admissibility condition (2.19) is satisfied. This follows from

$$
\begin{aligned}
|\phi(u, v, w ; z)| & =\left|1+\frac{\lambda k+1+M e^{i \theta}}{1+M e^{i \theta}} M e^{i \theta}-1-M e^{i \theta}\right| \\
& =\frac{\lambda M k}{\mid 1+M e^{i \theta \mid}} \geq \frac{\lambda M}{1+M}
\end{aligned}
$$

for $z \in U, \theta \in \mathbb{R}, \lambda>0$ and $k \geq 1$. Hence the result is easily deduced from Corollary 6 . 
3. Superordination results of the linear operator $D_{\lambda, p}^{n}$. In this section we obtain differential superordination for functions associated with the linear operator $D_{\lambda, p}^{n}$. For this purpose the class of admissible functions is given in the following definition.

Definition 7. Let $\Omega$ be a set in $\mathbb{C}$ and $q(z) \in H$ with $z q^{\prime}(z) \neq 0$. The class of admissible functions $\Phi_{D}^{\prime}[\Omega, q]$ consists of those functions $\phi: \mathbb{C}^{3} \times \bar{U} \rightarrow \mathbb{C}$ which satisfy the admissibility condition:

$$
\phi(u, v, w ; \zeta) \in \Omega
$$

whenever $u=q(z), v=\frac{\lambda z q^{\prime}(z)+m q(z)}{m}$,

$$
\Re\left\{\frac{w-2 v+u}{\lambda(v-u)}\right\} \leq \frac{1}{m} \Re\left\{1+\frac{z q^{\prime \prime}(z)}{q^{\prime}(z)}\right\},
$$

where $z \in U, \zeta \in \partial U$, and $m \geq 1$.

Theorem 7. Let $\phi \in \Phi_{D}^{\prime}[\Omega, q]$. If $f(z) \in \sum_{p}, z^{p} D_{\lambda, p}^{n} f(z) \in Q_{1}$ and

$$
\phi\left(z^{p} D_{\lambda, p}^{n} f(z), z^{p} D_{\lambda, p}^{n+1} f(z), z^{p} D_{\lambda, p}^{n+2} f(z) ; z\right)
$$

is univalent in $U$, then

$$
\Omega \subset\left\{\phi\left(z^{p} D_{\lambda, p}^{n} f(z), z^{p} D_{\lambda, p}^{n+1} f(z), z^{p} D_{\lambda, p}^{n+2} f(z) ; z\right): z \in U\right\}
$$

implies

$$
q(z) \prec z^{p} D_{\lambda, p}^{n} f(z) .
$$

Proof. Let $g(z)$ be defined by $(2.2)$ and $\psi$ by (2.6). Since $\phi \in \Phi_{D}^{\prime}[\Omega, q]$, (2.7) and (3.1) yield

$$
\Omega \subset\left\{\psi\left(g(z), z g^{\prime}(z), z^{2} g^{\prime \prime}(z) ; z\right): z \in U\right\} .
$$

From (2.6), we see that the admissibility condition for $\phi \in \Phi_{D}^{\prime}[\Omega, q]$ is equivalent to the admissibility condition for $\psi$ as given in Definition 2 . Hence $\psi \in \Psi^{\prime}[\Omega, q]$, and by Lemma 2 ,

$$
q(z) \prec g(z) \quad \text { or } \quad q(z) \prec z^{p} D_{\lambda, p}^{n} f(z) .
$$

If $\Omega \neq \mathbb{C}$ is a simply connected domain, then $\Omega=h(U)$ for some conformal mapping $h(z)$ of $U$ onto $\Omega$ and the class $\Phi_{D}^{\prime}[h(U), q]$ is written as $\Phi_{D}^{\prime}[h, q]$.

Proceeding similarly as in the previous section, we establish the following result as an immediate consequence of Theorem 7 .

Theorem 8. Let $q(z) \in H, h(z)$ be analytic in $U$ and $\phi \in \Phi_{D}^{\prime}[h, q]$. If $f(z) \in \sum_{p}, z^{p} D_{\lambda, p}^{n} f(z) \in Q_{1}$ and

$$
\phi\left(z^{p} D_{\lambda, p}^{n} f(z), z^{p} D_{\lambda, p}^{n+1} f(z), z^{p} D_{\lambda, p}^{n+2} f(z) ; z\right)
$$


is univalent in $U$, then

$$
h(z) \prec \phi\left(z^{p} D_{\lambda, p}^{n} f(z), z^{p} D_{\lambda, p}^{n+1} f(z), z^{p} D_{\lambda, p}^{n+2} f(z) ; z\right)
$$

implies

$$
q(z) \prec z^{p} D_{\lambda, p}^{n} f(z) .
$$

Theorems 7 and 8 can only be used to obtain subordinants of differential superordination of the form (3.1) or (3.2).

The following theorem proves the existence of the best subordinant of (3.2) for an appropriate $\phi$.

Theorem 9. Let $h(z)$ be analytic in $U$ and $\phi: \mathbb{C}^{3} \times \bar{U} \rightarrow \mathbb{C}$. Suppose that the differential equation

$$
\phi\left(g(z), \lambda z g^{\prime}(z)+g(z), \lambda^{2} z^{2} g^{\prime \prime}(z)+\lambda(2+\lambda) z g^{\prime}(z)+g(z) ; z\right)=h(z)
$$

has a solution $q(z) \in Q_{1}$. If $\phi \in \Phi_{D}^{\prime}[h, q], f(z) \in \sum_{p}, z^{p} D_{\lambda, p}^{n} f(z) \in Q_{1}$ and

$$
\phi\left(z^{p} D_{\lambda, p}^{n} f(z), z^{p} D_{\lambda, p}^{n+1} f(z), z^{p} D_{\lambda, p}^{n+2} f(z) ; z\right)
$$

is univalent in $U$, then

$$
h(z) \prec \phi\left(z^{p} D_{\lambda, p}^{n} f(z), z^{p} D_{\lambda, p}^{n+1} f(z), z^{p} D_{\lambda, p}^{n+2} f(z) ; z\right)
$$

implies

$$
q(z) \prec z^{p} D_{\lambda, p}^{n} f(z)
$$

and $q(z)$ is the best subordinant.

Proof. The proof is similar to the proof of Theorem 4 and is therefore omitted.

Combining Theorems 2 and 8, we obtain the following sandwich-type theorem.

Corollary 9. Let $h_{1}(z)$ and $q_{1}(z)$ be analytic functions in $U, h_{2}(z)$ be univalent function in $U, q_{2}(z) \in Q_{1}$ with $q_{1}(0)=q_{2}(0)=1$ and $\phi \in$ $\Phi_{D}\left[h_{2}, q_{2}\right] \cap \Phi_{D}^{\prime}\left[h_{1}, q_{1}\right]$. If $f(z) \in \sum_{p}, z^{p} D_{\lambda, p}^{n} f(z) \in H \cap Q_{1}$ and

$$
\phi\left(z^{p} D_{\lambda, p}^{n} f(z), z^{p} D_{\lambda, p}^{n+1} f(z), z^{p} D_{\lambda, p}^{n+2} f(z) ; z\right)
$$

is univalent in $U$, then

$$
h_{1}(z) \prec \phi\left(z^{p} D_{\lambda, p}^{n} f(z), z^{p} D_{\lambda, p}^{n+1} f(z), z^{p} D_{\lambda, p}^{n+2} f(z) ; z\right) \prec h_{2}(z)
$$

implies

$$
q_{1}(z) \prec z^{p} D_{\lambda, p}^{n} f(z) \prec q_{2}(z) .
$$


Definition 8. Let $\Omega$ be a set in $\mathbb{C}$ and $q(z) \in H$ with $z q^{\prime}(z) \neq 0$. The class of admissible functions $\Phi_{D, 1}^{\prime}[\Omega, q]$ consists of those functions $\phi: \mathbb{C}^{3} \times \bar{U} \rightarrow \mathbb{C}$ which satisfy the admissibility condition:

$$
\phi(u, v, w ; \zeta) \in \Omega
$$

whenever $u=q(z), v=q(z)+\frac{\lambda \zeta q^{\prime}(z)}{m q(z)}(q(z) \neq 0)$,

$$
\Re\left\{\frac{v w-3 u v+2 u^{2}}{\lambda(v-u)}\right\} \leq \frac{1}{m} \Re\left\{1+\frac{z q^{\prime \prime}(z)}{q^{\prime}(z)}\right\},
$$

where $z \in U, \zeta \in \partial U$ and $m \geq 1$.

Now we will give the dual result of Theorem 5 for differential superordination.

Theorem 10. Let $\phi \in \Phi_{D, 1}^{\prime}[\Omega, q]$. If $f(z) \in \sum_{p}, \frac{D_{\lambda, p}^{n+1} f(z)}{D_{\lambda, p}^{n} f(z)} \in Q_{1}$ and

$$
\phi\left(\frac{D_{\lambda, p}^{n+1} f(z)}{D_{\lambda, p}^{n} f(z)}, \frac{D_{\lambda, p}^{n+2} f(z)}{D_{\lambda, p}^{n+1} f(z)}, \frac{D_{\lambda, p}^{n+3} f(z)}{D_{\lambda, p}^{n+2} f(z)} ; z\right)
$$

is univalent in $U$, then

$$
\Omega \subset\left\{\phi\left(\frac{D_{\lambda, p}^{n+1} f(z)}{D_{\lambda, p}^{n} f(z)}, \frac{D_{\lambda, p}^{n+2} f(z)}{D_{\lambda, p}^{n+1} f(z)}, \frac{D_{\lambda, p}^{n+3} f(z)}{D_{\lambda, p}^{n+2} f(z)} ; z\right): z \in U\right\}
$$

implies

$$
q(z) \prec \frac{D_{\lambda, p}^{n+1} f(z)}{D_{\lambda, p}^{n} f(z)} .
$$

Proof. Let $g(z)$ be defined by (2.12) and $\psi$ by (2.17). Since $\phi \in \Phi_{D, 1}^{\prime}[\Omega, q]$, it follows from (2.18) and (3.3) that

$$
\Omega \subset\left\{\psi\left(g(z), z g^{\prime}(z), z^{2} g^{\prime \prime}(z) ; z\right): z \in U\right\} .
$$

From (2.17), we see that the admissibility condition for $\phi \in \Phi_{D, 1}^{\prime}[\Omega, q]$ is equivalent to the admissibility condition for $\psi$ as given in Definition 2 . Hence $\psi \in \Psi^{\prime}[\Omega, q]$, and by Lemma 2 ,

$$
q(z) \prec g(z) \quad \text { or } \quad q(z) \prec \frac{D_{\lambda, p}^{n+1} f(z)}{D_{\lambda, p}^{n} f(z)} .
$$

If $\Omega \neq \mathbb{C}$ is a simply connected domain and $\Omega=h(U)$ for some conformal mapping $h(z)$ of $U$ onto $\Omega$, then the class $\Phi_{D, 1}^{\prime}[h(U), q]$ is written as $\Phi_{D, 1}^{\prime}[h, q]$.

Proceeding similarly as in the previous section, we establish the following result as an immediate consequence of Theorem 10. 
Theorem 11. Let $q(z) \in H, h(z)$ be analytic in $U$ and $\phi \in \Phi_{D, 1}^{\prime}[h, q]$. If $f(z) \in \sum_{p}, \frac{D_{\lambda, p}^{n+1} f(z)}{D_{\lambda, p}^{n} f(z)} \in Q_{1}$ and

$$
\phi\left(\frac{D_{\lambda, p}^{n+1} f(z)}{D_{\lambda, p}^{n} f(z)}, \frac{D_{\lambda, p}^{n+2} f(z)}{D_{\lambda, p}^{n+1} f(z)}, \frac{D_{\lambda, p}^{n+3} f(z)}{D_{\lambda, p}^{n+2} f(z)} ; z\right)
$$

is univalent in $U$, then

$$
h(z) \prec \phi\left(\frac{D_{\lambda, p}^{n+1} f(z)}{D_{\lambda, p}^{n} f(z)}, \frac{D_{\lambda, p}^{n+2} f(z)}{D_{\lambda, p}^{n+1} f(z)}, \frac{D_{\lambda, p}^{n+3} f(z)}{D_{\lambda, p}^{n+2} f(z)} ; z\right)
$$

implies

$$
q(z) \prec \frac{D_{\lambda, p}^{n+1} f(z)}{D_{\lambda, p}^{n} f(z)} .
$$

Combining Theorems 6 and 11, we obtain the following sandwich-type theorem.

Corollary 10. Let $h_{1}(z)$ and $q_{1}(z)$ be analytic functions in $U, h_{2}(z)$ be univalent function in $U, q_{2}(z) \in Q_{1}$ with $q_{1}(0)=q_{2}(0)=1$ and $\phi \in$ $\Phi_{D, 1}\left[h_{2}, q_{2}\right] \cap \Phi_{D, 1}^{\prime}\left[h_{1}, q_{1}\right]$. If $f(z) \in \sum_{p}, \frac{D_{\lambda, p}^{n+1} f(z)}{D_{\lambda, p}^{n} f(z)} \in H \cap Q_{1}$ and

$$
\phi\left(\frac{D_{\lambda, p}^{n+1} f(z)}{D_{\lambda, p}^{n} f(z)}, \frac{D_{\lambda, p}^{n+2} f(z)}{D_{\lambda, p}^{n+1} f(z)}, \frac{D_{\lambda, p}^{n+3} f(z)}{D_{\lambda, p}^{n+2} f(z)} ; z\right)
$$

is univalent in $U$, then

$$
h_{1}(z) \prec \phi\left(\frac{D_{\lambda, p}^{n+1} f(z)}{D_{\lambda, p}^{n} f(z)}, \frac{D_{\lambda, p}^{n+2} f(z)}{D_{\lambda, p}^{n+1} f(z)}, \frac{D_{\lambda, p}^{n+3} f(z)}{D_{\lambda, p}^{n+2} f(z)} ; z\right) \prec h_{2}(z)
$$

implies

$$
q_{1}(z) \prec \frac{D_{\lambda, p}^{n+1} f(z)}{D_{\lambda, p}^{n} f(z)} \prec q_{2}(z) .
$$

Remark. Putting $\lambda=1$ in the above results, we obtain the similar results associated with the operator $D_{p}^{n}$.

\section{REFERENCES}

[1] Ali, R. M., Ravichandran, V. and Seenivasagan, N., Subordination and superordination of Liu-Srivastava linear operator on meromorphic functions, Bull. Malays. Math. Sci. Soc. 31, no. 2 (2008), 193-207.

[2] Aouf, M. K., Hossen, H. M., New criteria for meromorphic p-valent starlike functions, Tsukuba J. Math. 17 (1993), 481-486.

[3] Liu, J.-L., Owa, S., Certain meromorphic p-valent functions, Taiwanese J. Math. 2, no. 1 (1998), 107-110. 
[4] Liu, J.-L., Srivastava, H. M., Subclasses of meromorphically multivalent functions associated with a certain linear operator, Math. Comput. Modelling 39, no. 1 (2004), $35-44$.

[5] Miller, S. S, Mocanu, P. T., Differential Subordinations: Theory and Applications, Series on Monographs and Textbooks in Pure and Applied Mathematics, Vol. 225, Marcel Dekker, New York and Basel, 2000.

[6] Miller, S. S, Mocanu, P. T., Subordinants of differential superordinations, Complex Var. Theory Appl. 48, no. 10 (2003), 815-826.

[7] Srivastava, H. M., Patel, J., Applications of differential subordinations to certain classes of meromorphically multivalent functions, J. Ineq. Pure Appl. Math. 6, no. 3 , art. 88 (2005), pp. 15.

[8] Uralegaddi, B. A., Somanatha, C., New criteria for meromorphic starlike univalent functions, Bull. Austral. Math. Soc. 43 (1991), 137-140.

M. K. Aouf

Department of Mathematics

Faculty of Science

Mansoura University

Mansoura 35516

Egypt

e-mail: mkaouf127@yahoo.com
T. M. Seoudy

Department of Mathematics

Faculty of Science

Fayoum University

Fayoum 63514

Egypt

e-mail: tmseoudy@gmail.com

Received July 7, 2009 